PART 8

SYMBIOTIC STARS 


\title{
SYMBIOTIC STARS
}

\author{
A. A. BOYARCHUK \\ Crimean Astrophysical Observatory, U.S.S.R.
}

\begin{abstract}
There are some arguments that the symbiotic stars are binary, where one component is a red giant and the other component is a small hot star which is exciting a nebula. The symbiotic stars belong to the old disc population. Probably, symbiotic stars are just such an evolutionary stage for double stars as planetary nebulae for single stars.
\end{abstract}

The term 'symbiotic stars' was first introduced by P. W. Merrill and now is used to designate stellar objects whose spectra represent a combination of absorption features characteristic of a low temperature star with emission lines of high excitation. Several dozens of such objects are known today.

The analysis of published lists of symbiotic stars (Bidelman, 1954; Payne-Gaposchkin, 1957; Boyarchuk, 1969; Wackerling, 1970) has shown that different authors understand the term 'symbiotic stars' in different ways. Thus it is first of all necessary to determine what objects are related to the symbiotic stars. Boyarchuk (1969) has given the following criteria:

(i) The absorption lines of late-type spectra (TiO bands, $\mathrm{Ca}$ I, Ca II and others) must be seen.

(ii) The emission lines of $\mathrm{He} \mathrm{II,} \mathrm{O}$ III or higher ionized atoms must be present. The widths of the emission lines do not exceed $100 \mathrm{~km} \mathrm{~s}^{-1}$.

(iii) The stellar brightness can vary with an amplitude of up to 3 mag. during several weeks.

The presence of emission lines of hydrogen and of once ionized metals in the spectrum is not a characteristic sign of symbiotic stars because many other types of variable stars also have these emission lines in their spectra.

Several reviews of symbiotic stars were published in the last ten years (Merrill, 1958; Payne-Gaposchkin, 1975; Sahade, 1960, 1965; Boyarchuk, 1969, 1970; Swings, 1970). Boyarchuk (1970) gives an extended bibliography.

\section{Light Variations}

The light variation is one of the most characteristic features of symbiotic stars. The light curves can be considered as a whole complex of small simultaneous flares. This circumstance has made it possible to call these stars 'nova-like stars'. It is necessary to mention that different stars have rather different light curves. Moreover, the character of the light curve varies strongly from time to time.

Figure 1 shows the light curve of $Z$ And. The light variations have a rather complicated character. There are times, when the stellar brightness changes very little, for instance during 1905-1913. On the other hand, the star flared up by $4^{m}$ in 1914 and 1939. Quite a few flares with smaller amplitude were observed in other years. In 1920 


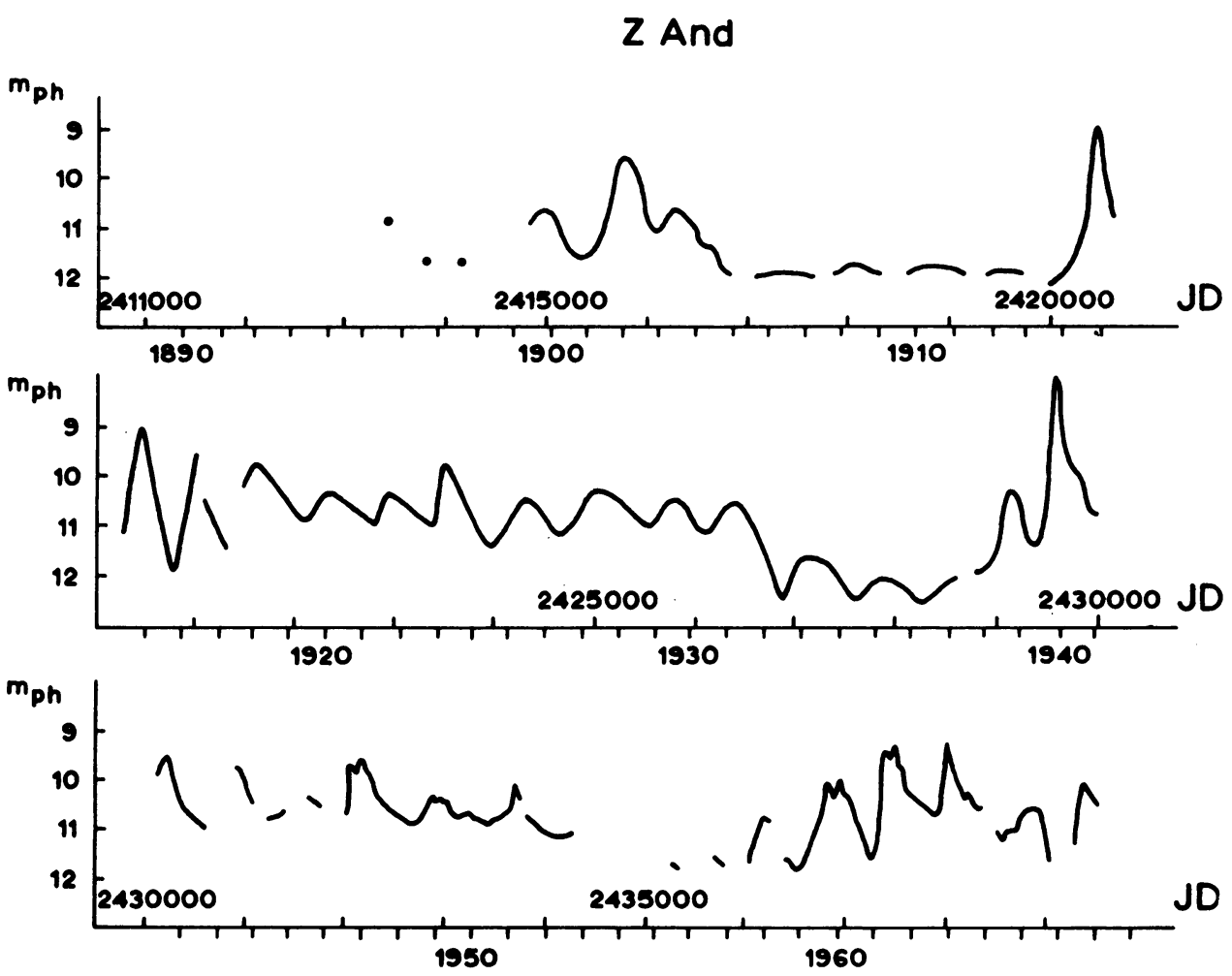

Fig. 1. The light curve of $Z$ And.

1931 the light variations had a quasi-periodic character with a period of $\sim 714^{d}$. Many symbiotic stars have similar light curves.

Photoelectric observations of the light variation of symbiotic stars (Belyakina, $1965,1967)$ have shown that many of them show rapid non-periodic light variations with small amplitudes. The largest short-time variations have been observed in the ultraviolet. Figure 2 shows the rapid light variations of AG Dra.

The brightness variations of symbiotic stars are accompanied by colour variations. As a rule the $B-V$ colour increases and the $U-B$ colour decreases with decreasing stellar brightness, i.e. the star becomes redder and the ultraviolet excess increases.

A periodic light variation with a constant amplitude has been discovered by Belyakina (1968) for AG Peg. The period of light variation is $\simeq 800^{d}$ and the amplitudes for different colours are: $\Delta U=1^{m} 1, \Delta B=0^{m} \cdot 7$ and $\Delta V=0^{m} \cdot 4$.

\section{Spectral Variations}

The character of symbiotic star spectra also changes with the light variations. Figure 3 shows the intensity tracings of spectrograms of AX Per taken in 1964 at $m_{\mathrm{ph}}=13.5$, and in 1965 , at $m_{\mathrm{ph}}=12^{m} .8$. We can see that the intensities of the [Fe VII] and He II lines 
SYMBIOTIC STARS

379

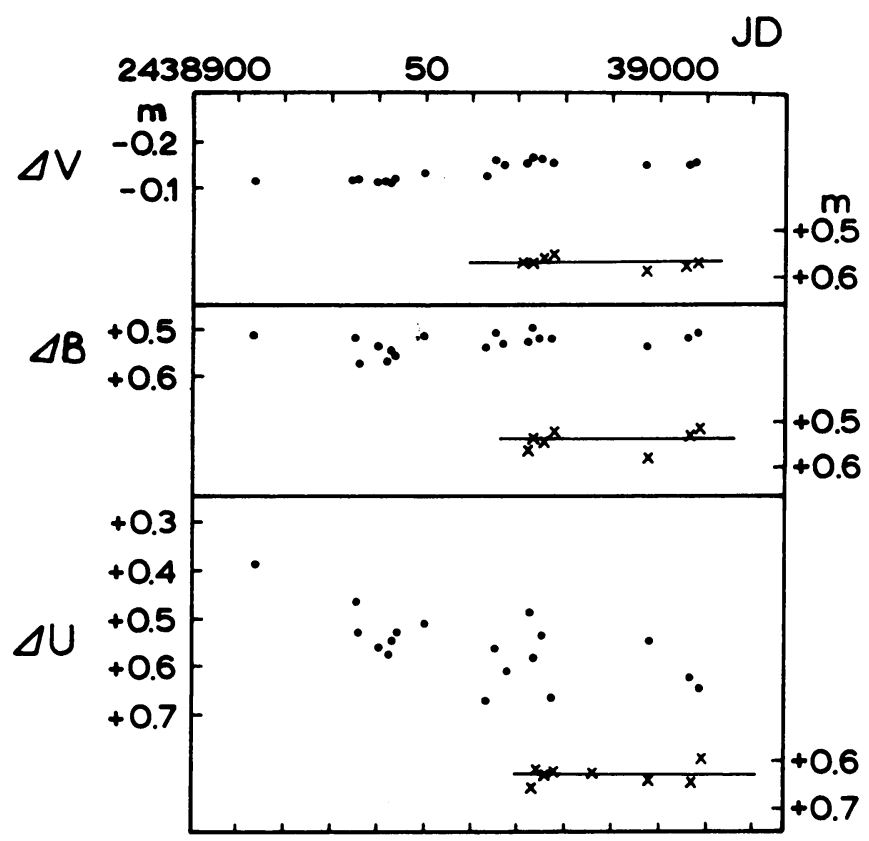

Fig. 2. The rapid light variations of AG Bra. The dots correspond to the brightness difference between the symbiotic star and the comparison star. The crosses correspond to the brightness difference between two comparison stars.

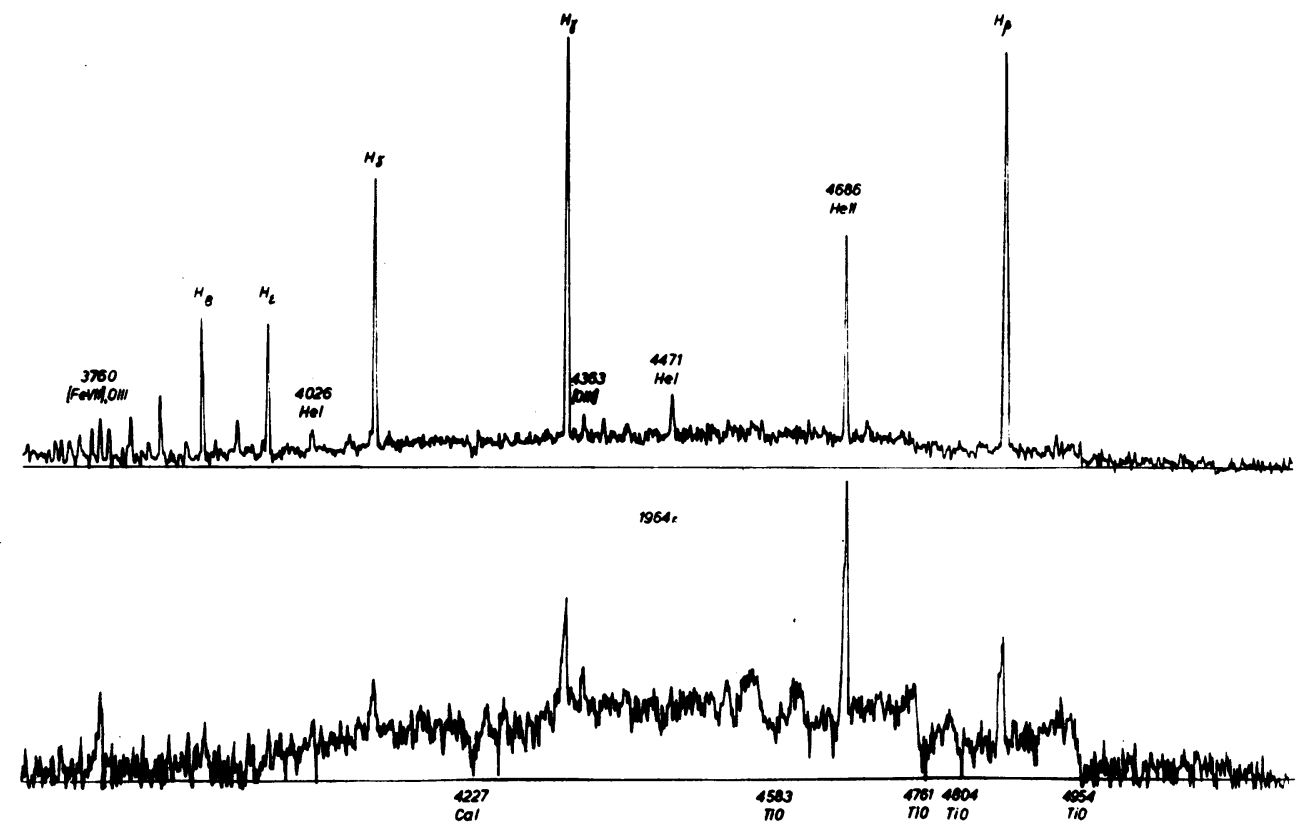

Fig. 3. The tracings of spectra of AX Per, taken in $1964\left(m_{\mathrm{ph}}=13.5\right)$ and in $1965\left(m_{\mathrm{ph}}=12.8\right)$.

https://doi.org/10.1017/S0074180900010524 Published online by Cambridge University Press 
decreased appreciably in comparison with those of hydrogen when the stellar brightness increased by $1 \mathrm{mag}$. At the same time, the intensity of the $\mathrm{TiO}$ bands strongly decreased.

Swings and Struve (1941) recognized that, during the large flare of $Z$ And in 1939, its spectrum underwent great changes. Late-type absorption features as well as highly excited emission lines disappeared completely. But absorption lines usually observed in the spectra of A0-A2 type stars were seen. The hydrogen and neutral helium emission lines had absorption components. At that time, the spectrum of $Z$ And was very similar to that of P Cyg-type stars. Late-type absorption features and highly excited emission lines appeared when the stellar brightness become fainter. Similar changes in the spectrum took place during the large flare of Z And in 1961 and of AX Per in 1955 (Gauzit, 1955).

The energy distributions in the continua of symbiotic stars vary in accord with colour variations. Figure 4 shows the observed energy distribution in the spectra of $Z$ And (solid lines) in 1960-1965. It is seen that the energy distribution becomes steeper corresponding to a later type spectrum and the Balmer jump increases when the star becomes fainter.

Let us suppose that the continua of symbiotic stars arise from three sources of radiation:

(i) a giant of type G-M,

(ii) a small hot star with $T_{\text {eff }} \simeq 10^{5} \mathrm{~K}$,

(iii) a nebula with $T_{\mathrm{e}} \simeq 17000 \mathrm{~K}$ and $n_{\mathrm{e}}>10^{6} \mathrm{~cm}^{-3}$.

The theoretical energy distributions in the spectra of these three sources are shown by open circles in Figure 4. The stellar magnitudes and other characteristices for each of the three sources of radiation are given in Table $I$ for different data. We can see that the agreement between the observed and the theoretical energy distributions is good enough.

TABLE I

\begin{tabular}{|c|c|c|c|c|c|c|}
\hline & $\begin{array}{l}1960 \\
\text { Oct. }\end{array}$ & $\begin{array}{l}1962 \\
\text { July }\end{array}$ & $\begin{array}{l}1963 \\
\text { Sept. }\end{array}$ & $\begin{array}{l}1964 \\
\text { Sept.-Oct. }\end{array}$ & $\begin{array}{l}1964 \\
\text { Dec. }\end{array}$ & $\begin{array}{l}1965 \\
\text { Oct. }\end{array}$ \\
\hline \multicolumn{7}{|l|}{$m_{\mathrm{ph}}$} \\
\hline $\begin{array}{l}\text { Z And } \\
\text { M2 III } \\
\text { Nebula } \\
\text { Hot comp. }\end{array}$ & $\begin{array}{l}11.65 \\
12.11 \\
14.00 \\
13.29\end{array}$ & $\begin{array}{l}10.75 \\
11.98 \\
13.25 \\
11.34\end{array}$ & $\begin{array}{l}10.55 \\
11.49 \\
12.90 \\
11.37\end{array}$ & $\begin{array}{l}11.00 \\
12.20 \\
12.86 \\
11.77\end{array}$ & $\begin{array}{l}10.65 \\
10.74 \\
12.78 \\
11.47\end{array}$ & $\begin{array}{l}11.70 \\
12.17 \\
13.68 \\
13.50\end{array}$ \\
\hline \multicolumn{7}{|l|}{ Hot comp. } \\
\hline $\begin{array}{l}T 10^{-3} \mathrm{~K} \\
M_{v} \\
R / R_{\odot}\end{array}$ & $\begin{array}{l}108 \\
2.82 \\
0.30\end{array}$ & $\begin{array}{l}70 \\
1.07 \\
0.70\end{array}$ & $\begin{array}{l}79 \\
1.57 \\
0.55\end{array}$ & $\begin{array}{l}96 \\
1.32 \\
0.60\end{array}$ & $\begin{array}{l}88 \\
1.55 \\
0.54\end{array}$ & $\begin{array}{r}141 \\
3.05 \\
0.26\end{array}$ \\
\hline \multicolumn{7}{|l|}{ Nebula } \\
\hline $\begin{array}{l}\lg n_{\mathrm{e}} \\
\mathfrak{M} \times 10^{-29} \mathrm{~g} \\
R \times 10^{-15} \mathrm{~cm}\end{array}$ & $\begin{array}{l}0.6 \\
0.8\end{array}$ & $\begin{array}{l}1.2 \\
0.9\end{array}$ & $\begin{array}{l}7.1 \\
1.0 \\
0.9\end{array}$ & $\begin{array}{l}7.3 \\
2.1 \\
1.1\end{array}$ & $\begin{array}{l}7.5 \\
1.3 \\
1.0\end{array}$ & $\begin{array}{l}0.9 \\
0.9\end{array}$ \\
\hline
\end{tabular}



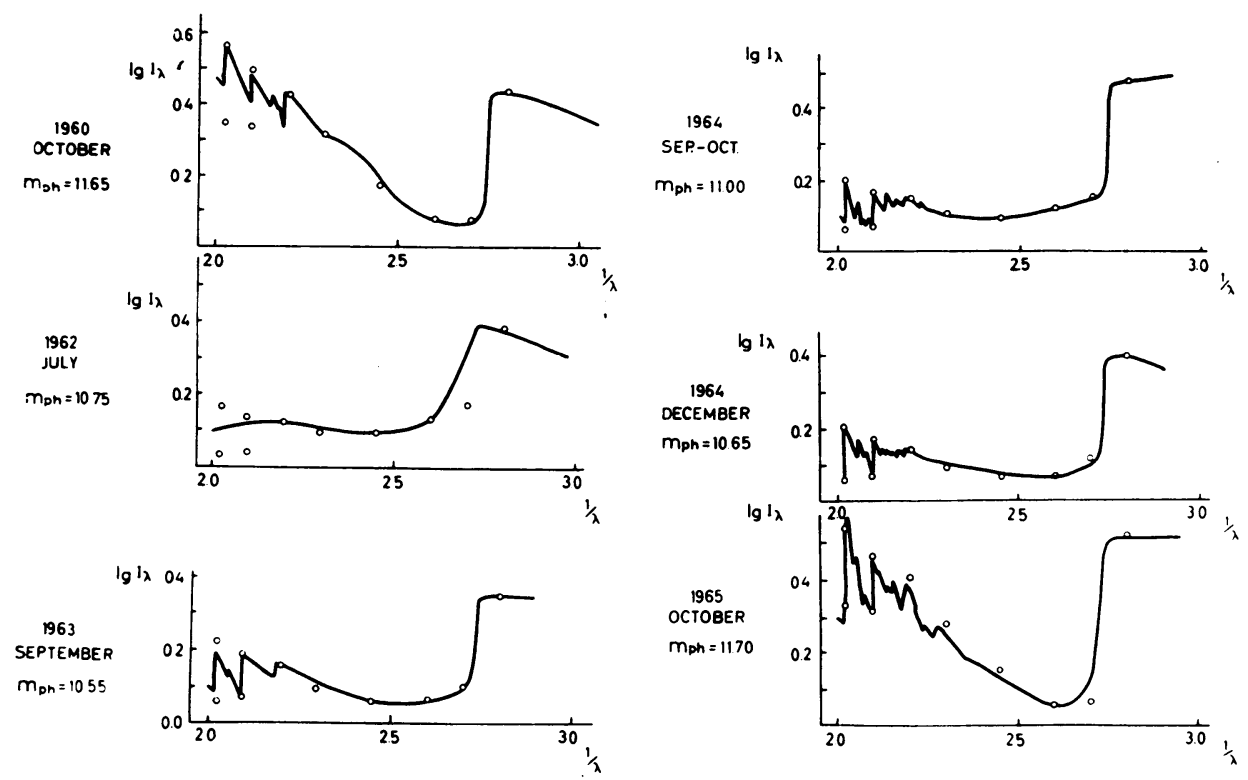

Fig. 4. The energy distribution in the continuum of $Z$ And. The solid lines represent observed distributions. The circles correspond to calculated intensities.

The spectral variations of symbiotic stars are dependent on variations of characteristics and the relative contribution of each source. The variations of the characteristics of the hot star play the most important rôle. Woolf (1969) has shown that the IR-fluxes at $11.5 \mu$ of symbiotic stars are in agreement with the three-sources model.

By using the above model, we can distinguish those lines which are formed mainly by one source. It is clear that absorption lines of late spectral type are produced in the atmosphere of the $\mathbf{M}$ giant as are the emission lines of neutral and ionized metals which are formed under the action of radiation from the hot star. The highly excited emission lines are formed in the atmosphere of the hot component or in the nebula surrounding it. Many emission lines, such as hydrogen lines, can be formed in each of the three sources and therefore, they are not characteristic of any one of the three sources.

If we consider displacements of lines belonging to the first two groups, we can see that they show a periodic variation for a majority of symbiotic stars. Some examples are shown on Figure 5. These periodic variations of radial velocity also confirm the proposed model for symbiotic stars. From the ratio of amplitudes of radial velocity variations, one can conclude that the mass of the $M$ giant is 3-5 times larger than that of the hot companion.

Cowley and Stencel (1973) have found that the mass ratio of the components of AG Peg is $\mathfrak{M}_{\text {cool }} / \mathfrak{M}_{\text {hot }} \sim 3$ from a solution of the radial velocity curve.

Thus, the photometric and spectral observations of symbiotic stars are in good agreement for such a model. Figure 6 shows a schematic model of a symbiotic star. 

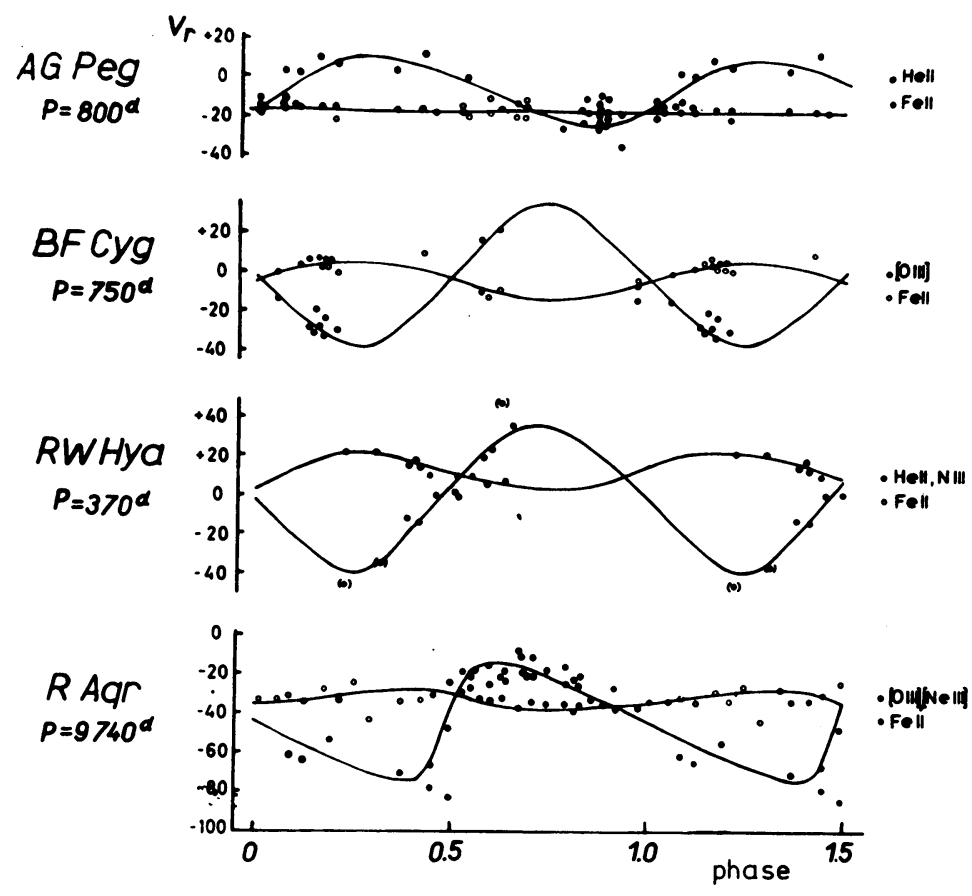

Fig. 5. The variation of radial velocities for some symbiotic stars.

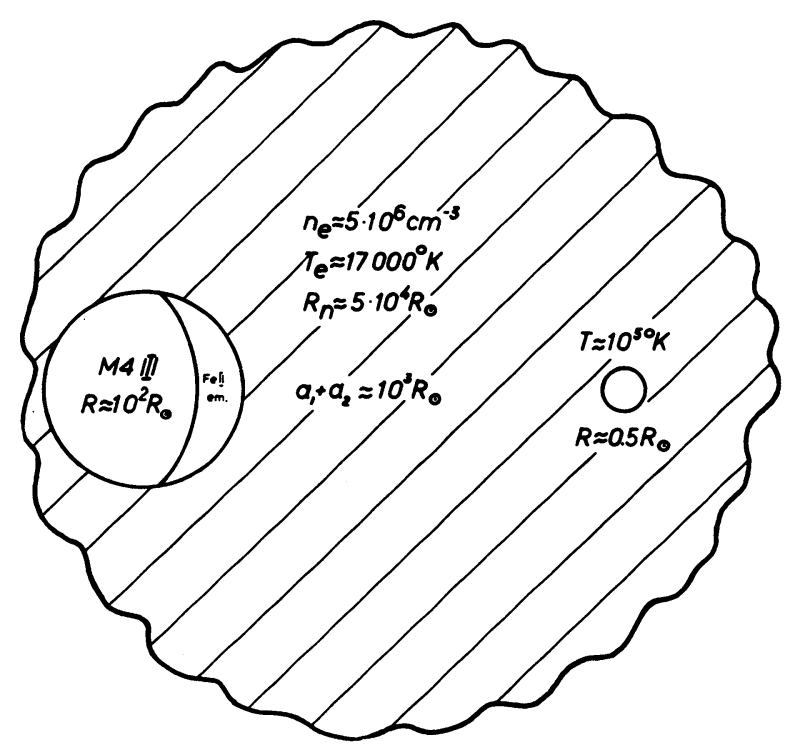

Fig. 6. The schematic model of a symbiotic star. 
If we suppose that the cool component is a normal giant of luminosity class III) then the hot component is located on the temperature-luminosity diagram below the main sequence as can be seen from Figure 7. The central stars of planetary nebulae, hot components of novae, etc. are located near this place too. Perhaps the nonstability of all these objects has a common nature.

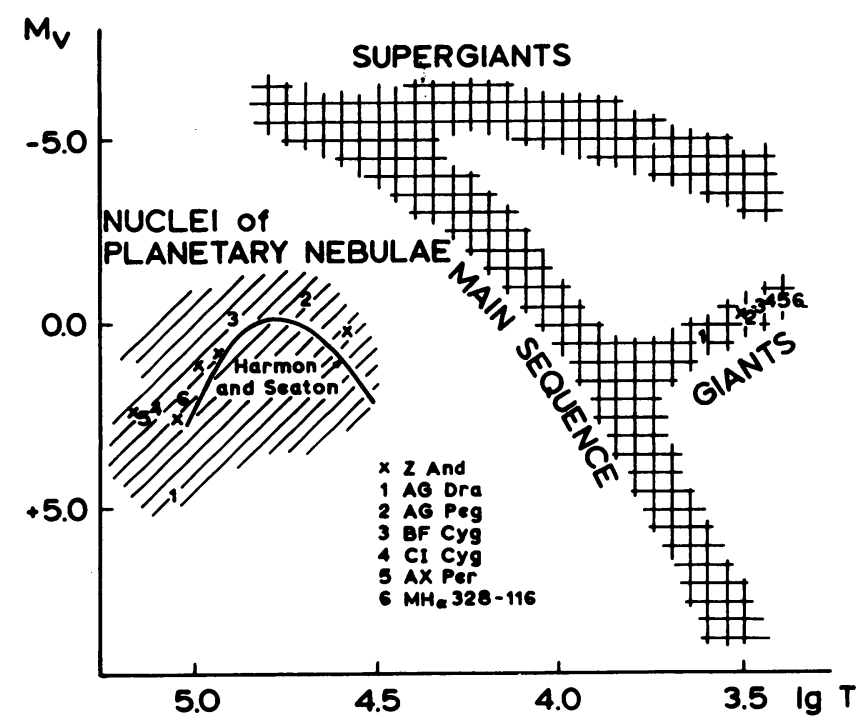

Fig. 7. The position of symbiotic stars on the temperature-luminosity diagram.

\section{The Space Distributions}

At the present time we know about 50 stars which can be considered with different degrees of probability as symbiotic stars. Such a small number of stars is not large enough for serious statistical considerations and we have now only a preliminary result (Boyarchuk, 1970).

First of all, it is necessary to point out that no known symbiotic star is a member of any cluster.

Figure 8 shows the apparent distribution of symbiotic stars in galactic coordinates. In the same figure is shown the distribution of planetary nebulae.

We can obtain the distribution of symbiotic stars in the galactic plane and in the $z$-coordinate if we assume that the luminosities of the cool components are close to those of normal giants of the same spectral type and that the cool component contributes one half of the total radiation from the symbiotic star in the system band. Figures 9 and 10 show the distribution of symbiotic stars in the galactic plane and that in the $z$-coordinate respectively.

Figures 8-10 show that the symbiotic stars and the planetary nebulae have very similar space distributions. Both groups have an appreciable concentration in the 

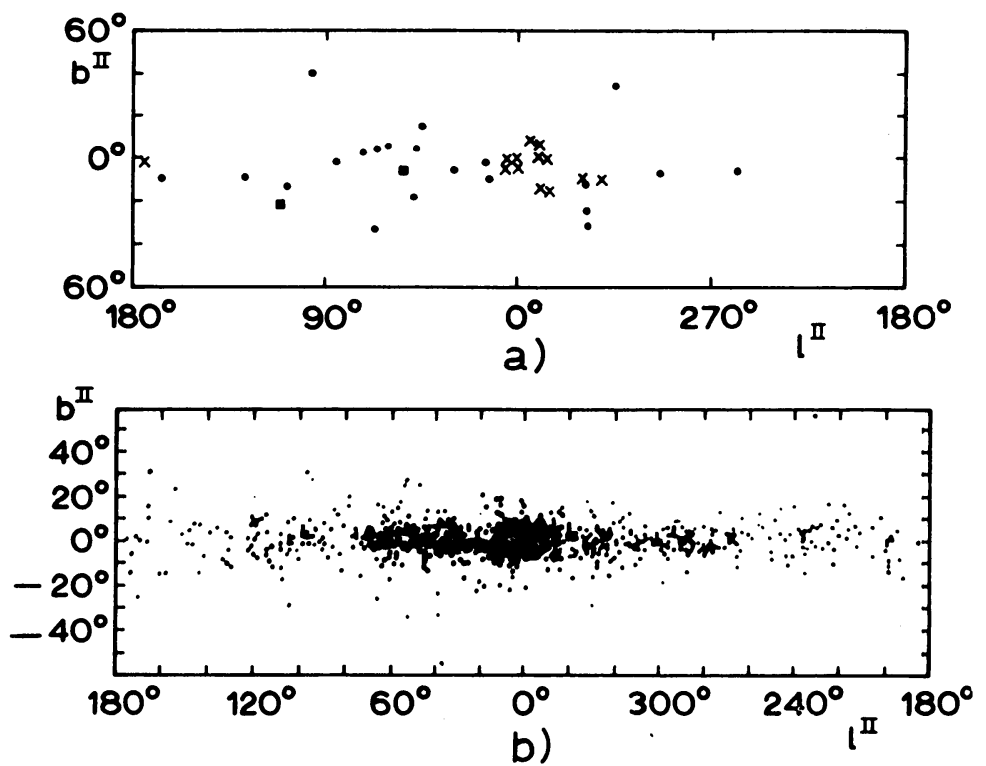

Fig. 8. The apparent distribution of symbiotic stars (a) and that of planetary nebulae (b). In Figure 8a the dots correspond to symbiotic stars, the crosses to probably symbiotic stars.

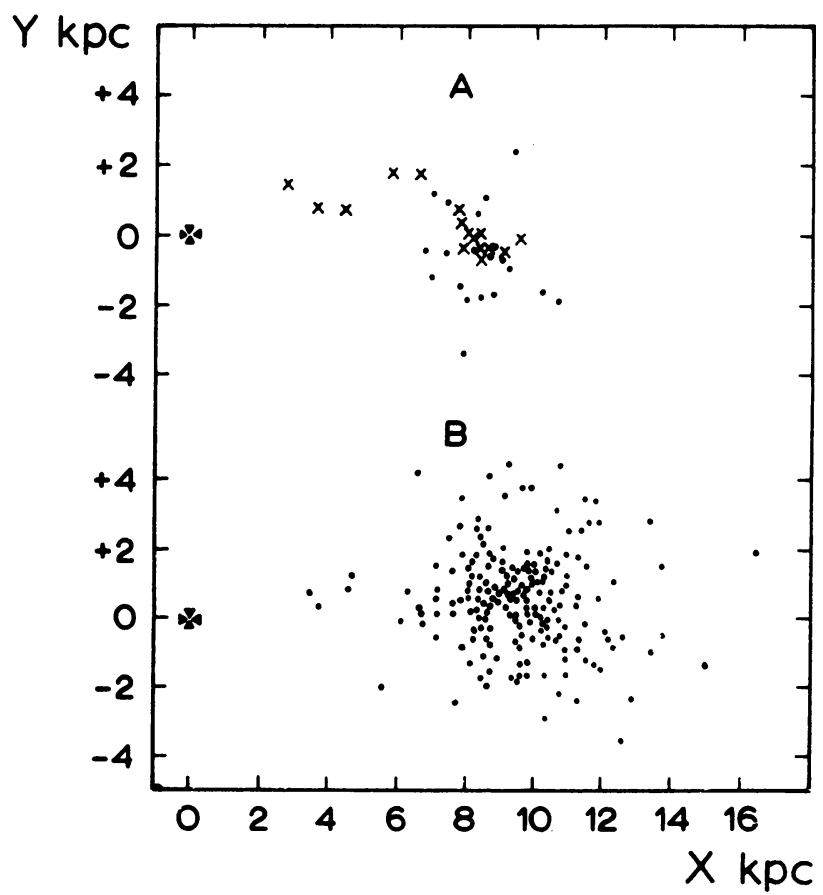

Fig. 9. The distribution of symbiotic stars and planetary nebulae in the galactic plane. The symbols are the same as in Figure 8. 


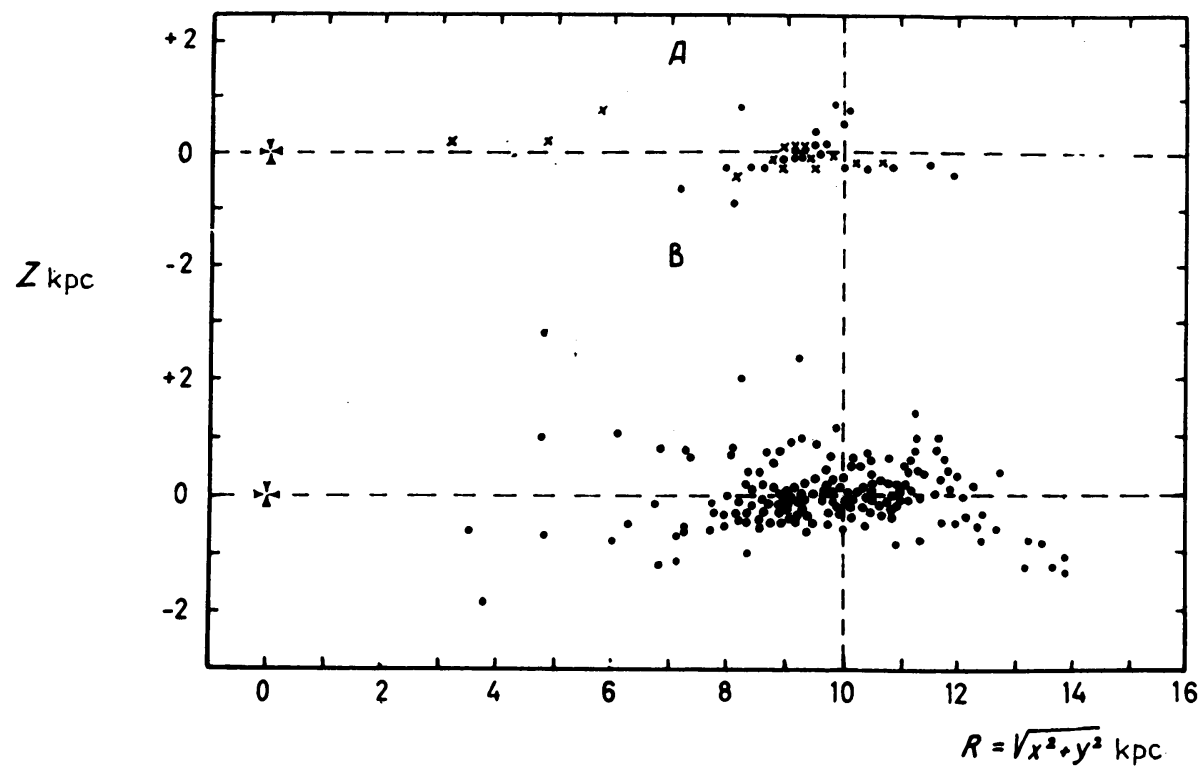

Fig. 10. The distribution of symbiotic stars and planetary nebulae in the Z-coordinate. The symbols are the same as in Figure 8.

galactic plane and show no concentration toward the galactic centre. One can conclude that the symbiotic stars belong to the old disc population.

We can estimate the total number of symbiotic stars in the Galaxy. Fourteen symbiotic stars are located within $2 \mathrm{kps}$ of the Sun. If symbiotic stars are distributed homogeneously in the galactic plane then the total number of symbiotic stars will be equal to $\sim 10^{3}$. Of course this value is rough, but any way, the symbiotic stars are rare objects.

In conclusion, we will consider some questions on the evolution of symbiotic stars.

It has been shown that symbiotic stars and nuclei of planetary nebulae are related objects in many respects. If one considers nuclei of planetary nebulae as an evolutionary stage of single stars of a certain mass, then the symbiotic stars can be considered as the same evolutionary stage for binaries in which the initially more massive components have evolved into the nuclei of planetary nebulae while the initially less massive components are still red giants.

It is well-known that about $40 \%$ of all young stars are binary. A binary nucleus is suspected only for the planetary nebula NGC 1514 . One can assume that the 'planetary nebula' stage for a double star is different from that of a single star. Under some conditions, this stage can have the form of a symbiotic star.

Recently, it was recognized that the BQ [] stars are similar to symbiotic stars in many respects. The designation BQ [ ] has been used to indicate a variety of hot stars with abnormal spectrum including the presence of forbidden lines (Wackerling, 1970). Ciatti, et al. (1974) have shown that the BQ [ ] stars are binaries: One component is a 
cool giant and the other is a hot star. There is also a nebula. The nebulae of BQ [ ] stars have a lower degree of excitation than those of symbiotic stars. Another difference between symbiotic stars and BQ [ ] stars is that the hot components of symbiotic stars have a higher temperature than those of BQ [] stars. Probably, the symbiotic stars and the $\mathrm{BQ}[]$ stars represent the same evolutionary stage but for stars of different masses.

\section{References}

Belyakina, T. S.: 1965, Izv. Krymsk. Astrophys. Obs. 33, 226.

Belyakina, T. S.: 1967, Izv. Krymsk. Astrophys. Obs. 38, 171.

Belyakina, T. S.: 1968, Astron. Zh. 45, $139=$ Soviet Astron. 12, 110.

Bidelman, W. P.: 1964, Astrophys. J. Suppl. 1, 175.

Boyarchuk, A. A.: 1969, in L. Detre (ed.), Non-Periodic Phenomena in Variable Stars, Academic Press, Budapest, p. 395.

Boyarchuk, A. A.: 1970, in A. Boyarchuk and R. Gershberg (eds.), Eruptive Stars, Nauka, Moscow, p. 113.

Ciatti, F., D'Odorico, S. D., and Mammano, A.: 1974, Astron. Astrophys. 34, 181.

Cowley, A. and Stencel, R.: 1973, Astrophys. J. 184, 687.

Gauzit, J.: 1955, Ann. Astrophys. 18, 354.

Payne-Gaposchkin, C.: 1957, The Galactic Novae.

Sahade, G.: 1960, in J. L. Greenstein (ed.), Stellar Atmospheres, Chicago, p. 494.

Sahade, G.: 1965, Kl. Veröff. Bamberg 40, 140 (Colloquium on Variable Stars).

Swings, P.: 1970, in G. H. Herbig (ed.), Spectroscopic Astrophysics, Univ. of California Press, Berkeley, p. 189.

Swings, P. and Struve, O.: 1941, Astrophys. J. 93, 356.

Wackerling, L. R.: 1970, Mem. Roy. Astron. Soc. 73, 153.

Woolf, N. J.: 1969, Astrophys. J. 157, L37.

\section{DISCUSSION} H. Gursky: U Gem stars and novae are often described as novae, but only novae seem to explode.
Why?

A. A. Boyarchuk: It is my opinion that in the case of novae, the companion star fills and overflows its Roche lobe, whereas in U Gem and similar systems this is not the case; i.e., the companion star has not filled the Roche lobe.

$M$. Friedjung: Some people have very different models on symbiotic stars. Do these stars form a very inhomogeneous group?

A. A. Boyarchuk: It depends on the proper classification. If one keeps to the right classification of a symbiotic star consisting of two stars, a low-temperature component with absorption lines and another one with high-excitation emission lines, the group must be rather homogeneous.

J. P. Swings: I do not entirely agree that there are 'many similarities between BQ [ ] and symbiotic stars'. Let it suffice to say that:

(i) the excitation of the lines in BQ[ ] stars is lower than in symbiotics;

(ii) in most cases there is no evidence for the existence of a cool companion to $B Q[]$ stars;

(iii) only the BQ[ ] stars exhibit strong infrared excess. As demonstrated by Swings and Allen (Publ. Astron. Soc. Pacific 84 (1972), 523) on the basis of a survey covering about 50 stars, the majority of symbiotic (and VV Cephei) stars have near infrared continua closely resembling those of late-type stars, the two exceptions being RX Pup and V1016 Cyg.

\section{Reference}

Wackerling, L. R.: 1970, Mem. Roy. Astron. Soc. 73, 153 - BQ[] = B type stars with shell component present in spectrum; [ ] = forbidden lines. 
A. Mammano: Recently BQ[] stars with excitation as high as in symbiotic stars have been found, so that the only difference between the two classes is to be found in the infrared excess. When the red component will become a long period variable of the cygnid* class, it will provide more infrared excess, and the system will pass from the symbiotic to the BQ[ ] stage.

J. P. Swings: Dr Boyarchuk mentioned that symbiotic stars and planetary nebulae show many similarities, and said that planetary nebulae exhibited IR excess. This is true only for high density planetary nebulae, or compact (star-like) nebulae which show prominent infrared excesses (H-K index $\geqq 1 \mathrm{mag}$ ) due to thermal emission from dust. Such objects confirm the correlation between the existence of an infrared excess and the existence in their spectrum of forbidden emission lines of low excitation (i.e. [O I], [S II] and [Fe II]).

'Normal' planetaries exhibit IR fluxes attributable to free-free radiation, not to dust.

* Cygnid class $=$ NML Cyg-like stars. 